\title{
Grid Embedding of 4-Connected Plane Graphs*
}

\author{
$\mathrm{Xin} \mathrm{He}$ \\ Department of Computer Science, \\ State University of New York at Buffalo, \\ Buffalo, NY 14260, USA \\ xinhe@cs.buffalo.edu
}

\begin{abstract}
A straight line grid embedding of a plane graph $G$ is a drawing of $G$ such that the vertices are drawn at grid points and the edges are drawn as nonintersecting straight line segments. In this paper we show that if a 4-connected plane graph $G$ has at least four vertices on its external face, then $G$ can be embedded on a grid of size $W \times H$ such that $W+H \leq n, W \leq(n+3) / 2$ and $H \leq 2(n-1) / 3$, where $n$ is the number of vertices of $G$. Such an embedding can be computed in linear time.
\end{abstract}

\section{Introduction}

Let $G=(V, E)$ be a graph with $n$ vertices. We always assume $n \geq 3$ in this paper. $G$ is planar if it can be drawn on the plane such that the vertices are located at distinct points, and the edges are represented by nonintersecting curves joining their endpoints. A plane graph is a planar graph with a fixed plane embedding. A straight line grid embedding of a planar graph is a drawing where the vertices are located at distinct grid points, and the edges are represented by straight line segments. Such embeddings on reasonably small grids are very useful in visualizing planar graphs on graphic screens and have applications in CAD/CAM and Computer Graphics [6].

Wagner [24], Fáry [8], and Stein [22] independently showed that every planar graph has a straight line embedding. Since then, many embedding algorithms have been reported (e.g., [23], [2], and [16]). The earlier algorithms all suffer two serious drawbacks that render them useless in practice, as noted by de Fraysseix et al. [9]. First, they require high-precision real arithmetic relative to the size of the graph, and therefore cannot be used even for a graph of moderate size. Second, in the drawings produced by these algorithms, the ratios of the largest distance to the smallest distance between vertices

\footnotetext{
* This work was partially supported by National Science Foundation, Grant CCR-9205982.
} 


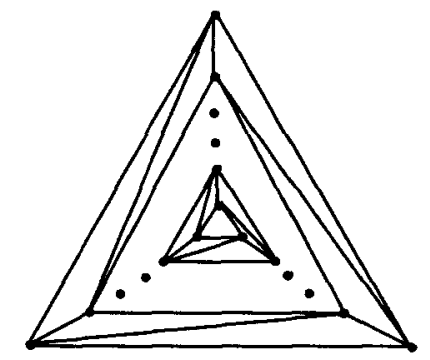

Fig. 1. A graph that requires a $(2 n / 3-1) \times(2 n / 3-1)$ grid.

are so large (exponential in $n$ ) that it is very difficult to view those drawings on graphic screens.

In view of these drawbacks, Rosenstiehl and Tarjan [17] posed the problem of computing a straight line embedding on a grid of polynomial size. Schnyder [19] proved that every planar graph has a straight line embedding on a $(2 n-4) \times(2 n-4)$ grid. Independently, de Fraysseix et al. showed that every planar graph has an embedding on a $(2 n-4) \times(n-2)$ grid, which can be computed in $O(n \log n)$ time [9], [10]. The running time of their algorithm was improved by Chrobak and Payne to $O(n)$ [5]. Schnyder further proved the existence of an embedding on an $(n-2) \times(n-2)$ grid [18] and gave an $O(n)$-time algorithm to compute such an embedding [20]. Schnyder's algorithm can be implemented in parallel in $O(\log n \log \log n)$ time with $n / \log n$ processors on a parallel random access machine [11]. It was shown in [12] that every 3-connected planar graph $G$ can be embedded on a $(2 n-4) \times(n-2)$ grid such that all internal faces of $G$ are convex polygons. The grid size of such embedding is reduced to $(n-2) \times(n-2)$ in [3], [13], and [21].

It has been observed that there exists a plane graph $G$, as shown in Fig. 1, such that the straight line grid embedding of $G$ requires a grid of size at least $(2 n / 3-1) \times(2 n / 3-1)$ [9]. Further, it was shown that for any straight line grid embedding of $G$, each dimension of the grid needs to be at least $\lfloor 2(n-1) / 3\rfloor$ even if the other dimension is allowed to be unbounded [4]. It has been conjectured that every planar graph can be embedded on a $2 n / 3 \times 2 n / 3$ grid. While this conjecture remains open, some progress has been made recently: Chrobak and Nakano showed that every planar graph has a straight line embedding on a $(\lfloor 2(n-1) / 3\rfloor) \times(4\lfloor 2(n-1) / 3\rfloor-1)$ grid [4].

For the straight line grid embedding problem, we can assume, without loss of generality, that all internal faces of $G$ are triangles. (If not, we can add dummy edges so that all internal faces of $G$ are triangles. After an embedding is obtained, the dummy edges can be removed.) If all internal faces of $G$ are triangles, it is called an internally triangulated plane graph. If the external face of $G$ is also a triangle, then $G$ is called a triangulated plane graph. From now on, we only consider internally triangulated plane graphs.

Given such a graph $G$, a separating triangle of $G$ is a triangle of $G$ whose removal disconnects $G$. Note that the graph $G$ shown in Fig. 1 consists of many levels of nested separating triangles. If $G$ is 4-connected, then $G$ cannot have separating triangles. (If $G$ has a separating triangle $\{x, y, z\}$, then $\{x, y, z\}$ would be a separating triple of $G$. 
This contradicts the 4-connectivity of $G$.) In this paper we show that if a 4-connected plane graph $G$ has at least four vertices on its external face, then the above mentioned $(2 n / 3-1) \times(2 n / 3-1)$ lower bound on grid size does not hold. We will prove:

Theorem 1.1. Let $G$ be an $n$ vertex internally triangulated 4-connected plane graph. If $G$ has at least four vertices on its external face, then $G$ has a straight line embedding on a $W \times H$ grid such that $W+H \leq n, W \leq(n+3) / 2$ and $H \leq 2(n-1) / 3$. Such an embedding can be computed in linear time.

This paper is organized as follows. In Section 2 we review some definitions and briefly describe a generic shift algorithm in [4], which is the basis of our algorithm. In Section 3 we present our algorithm. In Section 4 we prove Theorem 1.1. Section 5 concludes the paper.

\section{Preliminaries}

Most graph-theoretic definitions used here are standard (e.g., [1] and [7]). The embedding algorithms in [9], [10], [5], [4], [12], and [13] are all based on the following canonical ordering concept introduced in [9].

Definition 2.1. Let $G=(V, E)$ be a triangulated plane graph with $n$ vertices. Let $\pi=v_{1}, v_{2}, \ldots, v_{n}$ be an ordering of $V$ such that the edge $\left(v_{1}, v_{2}\right)$ is on the external face of $G$. Let $G_{k}$ be the subgraph of $G$ induced by $v_{1}, \ldots, v_{k}$ and $C_{k}$ be the external face of $G_{k} . \pi$ is called a canonical ordering of $G$ if the following conditions hold for each $k=3, \ldots, n$ :

(co1) $G_{k}$ is 2-connected and all internal faces of $G_{k}$ are triangles.

(co2) $C_{k}$ contains the edge $\left(v_{1}, v_{2}\right)$.

(co3) If $k<n$, then $v_{k+1}$ is in the external face of $G_{k}$ and all neighbors of $v_{k+1}$ in $G_{k}$ belongs to $C_{k}$.

(co4) If $k<n$, then $v_{k}$ has at least one neighbor $v_{j}$ with $j>k$.

Every triangulated plane graph has a canonical ordering [9], [10]. We use the symbol $\prec$ to denote the linear order of the canonical ordering. Namely, if $i<j$, then we write $v_{i} \prec v_{j}$. Note that condition (co4) is implied by condition (co3). For the sake of clarity, we make (co4) an explicit condition. Figure 2 shows a canonical ordering of a triangulated plane graph $G$.

By the contour of $G_{k}$ we mean its external face $C_{k}$ written as $C_{k}=\left(w_{1}=v_{1}, w_{2}, \ldots\right.$, $\left.w_{m}=v_{2}\right)$. For a given $k(3 \leq k \leq n-1)$, let $w_{p}, \ldots, w_{q}$ be the neighbors of $v=v_{k+1}$ in $C_{k}$. When we add $v$ to $G_{k}$, the leftmost edge $\left(w_{p}, v\right)$ and the rightmost edge $\left(v, w_{q}\right)$ become contour edges. We call $\left(w_{p}, v\right)$ a forward edge and $\left(v, w_{q}\right)$ a backward edge. All vertices and edges that disappear from the contour when we add $v$ are said to be covered by $v$. We call $v$ the cover vertex of $w_{j}$ for $p<j<q$. We call $w_{p}$ the first child of $v ; w_{p+1}$ the second child of $v \ldots$, and $w_{q}$ the last child of $v$. We denote $i n d_{v}\left(w_{i}\right)=i-p+1$ and call it the index of $w_{i}$ with respect to $v$. The in-degree $\mathrm{deg}^{-}(v)$ of $v$ is the number 


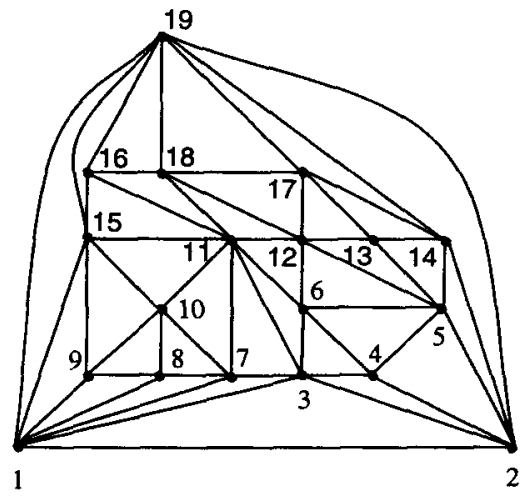

Fig. 2. A triangulated plane graph $G$ and its canonical ordering.

of children of $v$ in $C_{k}$, that is, $\mathrm{deg}^{-}(v)=q-p+1$. If $n \geq 4$, clearly $\mathrm{deg}^{-}\left(v_{k}\right) \geq 2$ for $4 \leq k \leq n-1$ and $\operatorname{deg}^{-}\left(v_{n}\right) \geq 3$.

A contour vertex $w_{i}(1<i<m)$ is called a valley vertex, if $w_{i-1} \succ w_{i} \prec w_{i+1}$; a peak vertex, if $w_{i-1} \prec w_{i} \succ w_{i+1}$. A vertex $v_{k+1} \neq v_{1}, v_{2}, v_{3}$ is called:

- Forward-oriented, if $w_{p} \prec w_{p+1} \prec \cdots \prec w_{q-1} \prec w_{q}$.

- Backward-oriented, if $w_{p} \succ w_{p+1} \succ \cdots \succ w_{q-1} \succ w_{q}$.

- Crossing-valley, if it covers a valley vertex $w_{r}(p<r<q)$.

- Crossing-peak, if it covers a peak vertex $w_{r}(p<r<q)$.

In particular, a vertex of in-degree 2 is forward-oriented (resp. backward-oriented) if and only if it covers a forward (resp. backward) edge. A vertex of in-degree $\geq 4$ can be both a crossing-valley and a crossing-peak vertex.

We next briefly describe a generic shift algorithm in [4] which is the basis of the embedding algorithms in [3], [4], [5], [9], [10], [12], [13], and our algorithm.

Given a triangulated plane graph $G=(V, E)$ with canonical ordering $\pi$, the genetic shift algorithm works as follows: We add vertices one at a time according to $\pi$. At each step, the contour $C_{k}$ satisfies certain contour invariants that involve restrictions on the slopes of contour edges. When adding a vertex $v_{k+1}$, we determine its location in the grid and, if necessary, shift some vertices of $G_{k}$ to the right in order to preserve the contour invariants. We have to determine which vertices of $G_{k}$ must be shifted to the right. This is done by maintaining a set $U(v)$ of vertices for each $v \in V . U(v)$ contains the vertices located "under" $v$ that need to be shifted whenever $v$ is shifted. The shift operation on a contour vertex $w_{j}$, denoted by shift $\left(w_{j}\right)$, is achieved by increasing the $x$-coordinate of each vertex $u \in \bigcup_{i=j}^{m} U\left(w_{i}\right)$ by 1 .

\section{Generic Shift Algorithm}

Initially, place $v_{1}, v_{2}, v_{3}$ at the points $(0,0),(2,0),(1,1)$, respectively. Let $U\left(v_{i}\right)=$ $\left\{v_{i}\right\}(1 \leq i \leq 3)$.

Suppose that $G_{k}(3 \leq k \leq n-1)$ has already been embedded, and we are about to add $v=v_{k+1}$. Let $C_{k}=\left(w_{1}, \ldots, w_{m}\right)$ be the contour of $G_{k}$ and let $w_{p}, \ldots, w_{q}$ be the 
children of $v$ in $C_{k}$. Define:

$$
U\left(v_{k+1}\right)=\left\{v_{k+1}\right\} \bigcup_{i=p+1}^{q-1} U\left(w_{i}\right) .
$$

Apply shift $\left(w_{i}\right)$ to some of $w_{1}, \ldots, w_{m}$ (possibly none), so that afterward there exists at least one grid point $\left(x^{\prime}, y^{\prime}\right)$ satisfying the following conditions, and that if $v$ is placed at $\left(x^{\prime}, y^{\prime}\right)$, then the contour invariants are preserved.

Definition 2.2. Generic shift conditions:

(gs1) $x\left(w_{p}\right) \leq x^{\prime} \leq x\left(w_{q}\right)$.

(gs2) $\left(x^{\prime}, y^{\prime}\right)$ is located above $C_{k}$ in the sense: the half line $\left\{\left(x^{\prime}, z\right) \mid z \geq y^{\prime}\right\}$ does not intersect $C_{k}$.

(gs3) All vertices $w_{p}, \ldots, w_{q}$ are visible from $\left(x^{\prime}, y^{\prime}\right)$. (Namely, the edges from $v$ to all $w_{p}, \ldots, w_{q}$ do not intersect each other, nor the edges in $C_{k}$.)

Place $v$ at an arbitrary point $\left(x^{\prime}, y^{\prime}\right)$ satisfying these conditions.

\section{End Generic Shift.}

The following theorem was implicit in [9], [10], and [5] and explicitly stated in [4].

Theorem 2.1. For all choices of shift operations and vertex coordinates, as long as (gs1), (gs2), and (gs3) are satisfied, the Generic Shift Algorithm produces a correct straight line grid embedding.

To use the generic shift algorithm, it is only necessary to specify the contour invariants and describe how to place the vertices so that both the contour invariants and the generic shift conditions are satisfied.

\section{Drawing Algorithm}

Our algorithm crucially depends on the following theorem which was proved in [14].

Theorem 3.1. Let $G=(V, E)$ be a 4-connected triangulated plane graph. Suppose that $\left\{v_{1}, v_{2}, v_{n}\right\}$ is the external face of $G$. Then $G$ has a canonical ordering $\pi$ satisfying conditions (co1), (co2), (co3), and the following condition:

(co4') If $k \leq n-2$, then $v_{k}$ has at least two neighbors $v_{j}$ with $v_{j} \succ v_{k}$. The vertex $v_{n-1}$ has one neighbor $v_{n}$ with $v_{n} \succ v_{n-1}$.

Moreover, $\pi$ can be computed in linear time.

The canonical ordering $\pi$ of $G$ in Theorem 3.1 has the following property.

Lemma 3.2. The vertex $v_{n}$ is the only crossing-peak vertex of $G$ with respect to $\pi$. 
Proof. Toward a contradiction, suppose $v_{k+1}(k+1<n)$ is a crossing-peak vertex with respect to $\pi$. Let $w_{p}, \ldots, w_{q}$ be the children of $v_{k+1}$ in $C_{k}$. Then $v_{k+1}$ covers a peak vertex $w_{r}(p<r<q)$. So $v_{k+1}$ is the only neighbor of $w_{r}$ with $w_{r} \prec v_{k+1}$. This contradicts condition $\left(\cot ^{\prime}\right)$.

Let $G=(V, E)$ be an $(n-1)$ vertex 4-connected internally triangulated plane graph with at least four vertices on its external face. In this section we present our algorithm for embedding $G$.

In order to apply Theorem 3.1 to $G$, we need to modify $G$ as follows. Add a new vertex $v_{n}$ in the external face $F$ of $G$ and connect $v_{n}$ to all vertices on $F$ such that $\left\{v_{1}, v_{2}, v_{n}\right\}$ is the external face. The resulting graph $G^{+}$is called the extended graph of $G$. Since $G$ is 4-connected, $G^{+}$is also 4-connected and hence satisfies the conditions in Theorem 3.1.

In the following, we discuss the embedding of $G^{+}$by using the canonical ordering $\pi$ of $G^{+}$satisfying the conditions in Theorem 3.1, with the understanding that the vertex $v_{n}$ needs not be embedded and all added edges will be removed after the embedding is obtained. Note that the number of vertices in the original graph $G$ is $n-1$.

Let $n_{\mathrm{f}}$ and $n_{\mathrm{b}}$ denote the number of forward- and backward-oriented vertices in $G^{+}$, among $v_{4}, \ldots, v_{n}$. Since $v_{n}$ is a peak vertex, we have $n_{\mathrm{f}}+n_{\mathrm{b}} \leq n-4$. Without loss of generality, we assume $n_{\mathrm{f}} \leq n_{\mathrm{b}}$. (If not, we "vertically flip" $G^{+}$and swap $v_{1}$ and $v_{2}$. A forward-oriented vertex in $G^{+}$is a backward-oriented vertex in the flipped graph and vice versa. Clearly, $\pi$ is a canonical ordering of the flipped graph and Theorem 3.1 still holds.)

Direct the edges of $G^{+}$as follows: Direct an edge $e=(u, v)$ from $u$ to $v$, if $u \prec v$. We say the resulting directed acyclic graph $\overline{G^{+}}$is derived from $\pi$. It is easy to see that the canonical ordering $\pi$ is a topological ordering of $\overline{G^{+}}$, and any topological ordering of $\overline{G^{+}}$is a canonical ordering of $G^{+}$.

When the generic shift algorithm is performed on $G^{+}$, if there is a directed path $P$ from a vertex $u$ to a vertex $v$ in $\overline{G^{+}}$, then $u$ must be drawn before $v$ (and vice versa). If no such $\boldsymbol{P}$ exists, however, the order in which $u$ and $v$ are drawn is irrelevant.

Our algorithm needs a special canonical ordering $\pi_{\text {left }}$ of $G^{+}$obtained as follows. The first three vertices of $\pi_{\text {left }}$ are $v_{1}, v_{2}, v_{3}$. Suppose the vertex $v_{k}$ of $\pi_{\text {left }}$ has been defined. Consider a vertex $v$ not in $G_{k}^{+}$. We say $u$ is an incoming neighbor of $v$, if there is an edge directed from $u$ to $v$ in $\frac{k}{G^{+}}$. If all incoming neighbors of $v$ are in the contour $C_{k}$ of $G_{k}^{+}, v$ is called a candidate vertex. The incoming neighbors of a candidate vertex form a contiguous interval in $C_{k}$. Define $v_{k+1}$ of $\pi_{\text {left }}$ to be the candidate vertex whose interval is the leftmost in $C_{k}$. This completes the description of $\pi_{\text {left }}$.

Clearly, $\pi_{\text {left }}$ is a topological ordering of $\overline{G^{+}}$and hence a canonical ordering of $G^{+}$. We use the symbol $\prec_{\text {left }}$ to denote the order defined by $\pi_{\text {left }}$. We call $\pi_{\text {left }}$ the leftmost canonical ordering of $G^{+}$derived from $\pi$. (Note that $\pi_{\text {left }}$ is different from the leftmost canonical ordering defined in [12] and [13]). Our algorithm uses $\pi_{\text {left }}$, which can be computed in linear time from $\pi$ by using the method in [15]. According to $\pi_{\text {left }}$, if there is more than one candidate vertex for $G_{k}^{+}$, the leftmost one is added first. Figure 3 shows the leftmost canonical ordering of the graph shown in Fig. 2.

We need the following concepts introduced in [4]. Each vertex $v\left(v \neq v_{1}, v_{2}\right)$ of $G^{+}$is classified as either stable or unstable. With each such vertex $v$, we associate a sequence 


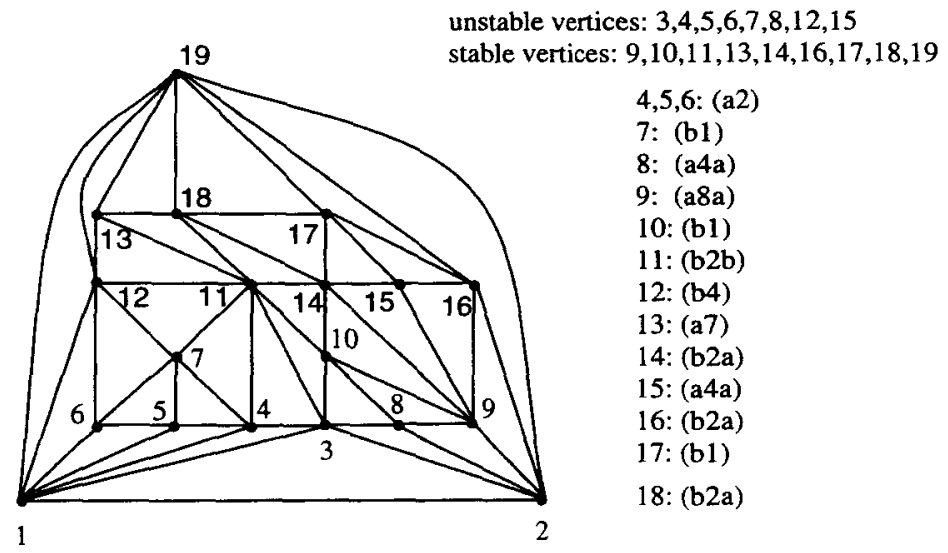

Fig. 3. The leftmost canonical ordering and the grid embedding of $G$.

$D C(v)$ of vertices called its domino chain, and a vertex $\operatorname{dom}(v)$ called its dominator. They are defined as follows.

For $v_{n}$, we define $D C\left(v_{n}\right)=\left(v_{n}\right), \operatorname{dom}\left(v_{n}\right)$ is undefined, and $v_{n}$ is stable. Consider $v=v_{k+1}(2 \leq k \leq n-2)$. Let $u$ be the leftmost child of $v$ in $C_{k}$, that is $i n d_{v}(u)=1$. Let $z$ be the vertex that covers the edge $(u, v)$. Such $z$ must exist because $v \neq v_{n}$. Then:

Definition 3.1. Domino chain and dominator:

(dc1) If $i n d_{z}(v)=2$, then $D C(v)=(v), \operatorname{dom}(v)=z$ and $v$ is unstable.

(dc2) If $\operatorname{ind}_{z}(v) \geq 4$, then $D C(v)=(v), \operatorname{dom}(v)=z$ and $v$ is stable.

(dc3) If $\operatorname{ind}_{z}(v)=3$ and $D C(z)=\left(z_{1}, \ldots, z_{i}, z\right)$, then $D C(v)=\left(z_{1}, \ldots, z_{i}, z, v\right)$ and $\operatorname{dom}(v)=\operatorname{dom}(z)$. Also, $v$ is stable if and only if $z$ is stable.

As in [4], an unstable vertex of in-degree 2 is called a room-shift vertex. The intuition is that a stable vertex can be placed above its leftmost child, saving one $x$-coordinate, while an unstable vertex must be placed at least one $x$-coordinate to the right of its leftmost child. If $v$ is a room-shift vertex, this can result in putting $v$ directly above its rightmost child $w$ and violating the contour invariants. In this case, we have to shift $w$ to the right in order to "make room" for $v$.

Example. In Fig. 3, the unstable and the stable vertices of $G$ are shown. $D C(14)=$ $(18,14) ; \operatorname{dom}(14)=19 . D C(9)=(10,9) ; \operatorname{dom}(9)=11 . D C(3)=(3) ; \operatorname{dom}(3)=4$.

Let $C_{k}=\left(w_{1}, \ldots, w_{m}\right)$ be the contour of $G_{k}^{+}$. The location of a vertex $w_{i}$ in the grid is referred to as the point $w_{i}$. A contour point $w_{i}$ is a peak point if $y\left(w_{i-1}\right)<y\left(w_{i}\right)$ and $y\left(w_{i}\right)>y\left(w_{i+1}\right) . w_{i}$ is a valley point if $y\left(w_{i-1}\right)>y\left(w_{i}\right)$ and $y\left(w_{i}\right)<y\left(w_{i+1}\right)$. The slope of a contour edge $e=\left(w_{i}, w_{i+1}\right)$ is denoted by slope $(e)$. We classify $e$ as follows:

- If $\operatorname{slope}(e)=0$, then $e$ is horizontal.

- If $0<\operatorname{slope}(e)<+\infty$, then $e$ is upward. 
- If slope $(e)=+\infty$ or $-\infty$, then $e$ is vertical.

- If $-\infty<\operatorname{slope}(e)<0$, then $e$ is downward.

We are now ready to describe our algorithm. It is a version of the generic shift algorithm. Our contour invariants are as follows:

Definition 3.2. Contour invariants:

(ci1) $x\left(w_{1}\right) \leq x\left(w_{2}\right) \leq \cdots \leq x\left(w_{m-1}\right) \leq x\left(w_{m}\right)$.

(ci2) Each forward edge $\left(w_{i-1}, w_{i}\right)$ is either horizontal, upward, or vertical. If $w_{i}$ is unstable, then $\left(w_{i-1}, w_{i}\right)$ must be horizontal or upward (thus $x\left(w_{i}\right) \geq$ $\left.x\left(w_{i-1}\right)+1\right)$.

(ci3) Each backward edge $\left(w_{i-1}, w_{i}\right)$ is either downward or horizontal (thus $x\left(w_{i}\right) \geq$ $\left.x\left(w_{i-1}\right)+1\right)$.

The following lemma was proved in [4]. We include its proof here for completeness.

Lemma 3.3. Let $w_{p}, \ldots, w_{q}$ be the children of the vertex $v=v_{k+1}$ in the contour $C_{k}$. Assume the contour invariants are satisfied. Then $x\left(w_{p}\right)<x\left(w_{p+1}\right)$. If $\operatorname{deg}^{-}(v)>2$ and $v$ is unstable, then $x\left(w_{p+1}\right)<x\left(w_{p+2}\right)$.

Proof. If $e_{1}=\left(w_{p}, w_{p+1}\right)$ is a backward edge, then $e_{1}$ cannot be vertical. If $e_{1}$ is a forward edge, then $w_{p+1}$ is unstable and $e_{1}$ cannot be vertical. In either case, we have $x\left(w_{p}\right)<x\left(w_{p+1}\right)$.

Suppose $\operatorname{deg}^{-}(v)>2$ and $v$ is unstable. If $e_{2}=\left(w_{p+1}, w_{p+2}\right)$ is a backward edge, then $e_{2}$ cannot be vertical. If $e_{2}$ is a forward edge, then since $v$ is unstable and $w_{p+2}$ is the third child of $v, w_{p+2}$ is unstable. Hence $e_{2}$ cannot be vertical. In either case, we have $x\left(w_{p+1}\right)<x\left(w_{p+2}\right)$.

Suppose we are about to add $v=v_{k+1}(3 \leq k \leq n-2)$. (Recall that the vertex $v_{n}$ is not embedded.) Let $C_{k}=\left(w_{1}, \ldots, w_{m}\right)$ be the contour of $G_{k}^{+}$and let $w_{p}, \ldots, w_{q}$ be the children of $v$ in $C_{k}$. Our algorithm must perform shift operation on some contour vertex (if necessary), and place $v$ at a point $(x(v), y(v))$ satisfying the generic shift conditions such that the contour invariants are preserved after $v$ is placed. This is ensured, as it can be easily checked, if $(x(v), y(v))$ satisfies the following:

Definition 3.3. Placement requirements:

(pr1) $x(v)<x\left(w_{q}\right)$. (This is required because the edge $\left(v, w_{q}\right)$ cannot be vertical).

(pr2) $x(v) \geq x\left(w_{p}\right)$, if $v$ is stable. $x(v) \geq x\left(w_{p}\right)+1$, if $v$ is unstable.

(pr3) $y(v) \geq \max \left\{y\left(w_{p}\right), y\left(w_{q}\right)\right\}$.

(pr4) $(x(v), y(v))$ is located above $C_{k}$, as defined in condition (gs2) in Definition 2.2.

(pr5) All vertices $w_{p}, \ldots, w_{q}$ are visible from $(x(v), y(v))$.

The placement rules for $v$ depend on the following factors:

- The in-degree of $v$. 
- $v$ is stable or unstable.

- $v$ is forward-oriented, backward-oriented, or crossing-valley.

- The edges covered by $v$ are horizontal, vertical, upward, or downward.

Let $W$ and $H$ denote the width and the height, respectively, of the current grid. In the following placement rules, we indicate how the values of $W$ and $H$ change after $v$ is placed. First consider the placement rules for $v=v_{k+1}$ when $\operatorname{deg}^{-}(v)=2$. Let $w_{p}$ and $w_{q}$ be the leftmost and the rightmost child of $v$. Note that the edge $\left(w_{p}, w_{q}\right)$ cannot be vertical by Lemma 3.3 .

Case (a1): $\operatorname{deg}^{-}(v)=2, v$ is unstable and forward-oriented, the edge $\left(w_{p}, w_{q}\right)$ is horizontal. $v$ is placed as in Fig. 4(a1): $x(v)=x\left(w_{p}\right)+1$ and $y(v)=y\left(w_{p}\right)+1$. If $x(v)=x\left(w_{q}\right)$, then perform shift $\left(w_{q}\right) . W$ is increased by at most $1 . H$ is increased by at most 1 .

Case (a2): $\operatorname{deg}^{-}(v)=2, v$ is unstable and forward-oriented, the edge $\left(w_{p}, w_{q}\right)$ is upward. $v$ is placed as in Fig. 4(a2): $x(v)=x\left(w_{p}\right)+1$ and $y(v)=y\left(w_{q}\right)$. If $x(v)=$ $x\left(w_{q}\right)$, then perform shift $\left(w_{q}\right) . W$ is increased by at most $1 . H$ is unchanged.

Case (a3): $\operatorname{deg}^{-}(v)=2, v$ is unstable and backward-oriented, the edge $\left(w_{p}, w_{q}\right)$ is horizontal. $v$ is placed as in Fig. 4(a3): $x(v)=x\left(w_{p}\right)+1$ and $y(v)=y\left(w_{p}\right)+1$. If $x(v)=x\left(w_{q}\right)$, then perform shift $\left(w_{q}\right) . W$ is increased by at most $1 . H$ is increased by at most 1 .

Case (a4a): $\operatorname{deg}^{-}(v)=2, v$ is unstable and backward-oriented, the edge $\left(w_{p}, w_{q}\right)$ is downward. $v$ is placed as in Fig. 4(a4a): $x(v)=x\left(w_{p}\right)+1$ and $y(v)=y\left(w_{p}\right)$. If $x(v)=x\left(w_{q}\right)$, then perform shift $\left(w_{q}\right) . W$ is increased by at most $1 . H$ is unchanged.

Case (a4b): Same as case (a4a). Figure 4(a4b) shows an alternative placement of $v$ : $x(v)=x\left(w_{p}\right)+1$ and $y(v)=y\left(w_{p}\right)+1$. If $x(v)=x\left(w_{q}\right)$, then perform shift $\left(w_{q}\right) . W$ is increased by at most $1 . H$ is increased by at most 1 .

Case (a5): $\mathrm{deg}^{-}(v)=2, v$ is stable and forward-oriented, the edge $\left(w_{p}, w_{q}\right)$ is horizontal. $v$ is placed as in Fig. 4(a5): $x(v)=x\left(w_{p}\right)$ and $y(v)=y\left(w_{p}\right)+1 . W$ is unchanged. $H$ is increased by at most 1 .

Case (a6): $\operatorname{deg}^{-}(v)=2, v$ is stable and forward-oriented, the edge $\left(w_{p}, w_{q}\right)$ is upward. $v$ is placed as in Fig. $4(\mathrm{a} 6): x(v)=x\left(w_{p}\right)$ and $y(v)=y\left(w_{q}\right) . W$ is unchanged. $H$ is unchanged.

Case (a7): $\operatorname{deg}^{-}(v)=2, v$ is stable and backward-oriented, the edge $\left(w_{p}, w_{q}\right)$ is horizontal. $v$ is placed as in Fig. 4(a7): $x(v)=x\left(w_{p}\right)$ and $y(v)=y\left(w_{p}\right)+1 . W$ is unchanged. $H$ is increased by at most 1 .

Case (a8a): $\operatorname{deg}^{-}(v)=2, v$ is stable and backward-oriented, the edge $\left(w_{p}, w_{q}\right)$ is downward. $v$ is placed as in Fig. 4(a8a): $x(v)=x\left(w_{p}\right)+1$ and $y(v)=y\left(w_{p}\right)$. If $x(v)=x\left(w_{q}\right)$, then perform shift $\left(w_{q}\right) . W$ is increased by at most $1 . H$ is unchanged.

Case (a8b): Same as case (a8a). Figure 4(a8b) shows an alternative placement of $v$ : $x(v)=x\left(w_{p}\right)$ and $y(v)=y\left(w_{p}\right)+1 . W$ is unchanged. $H$ is increased by at most 1 . 


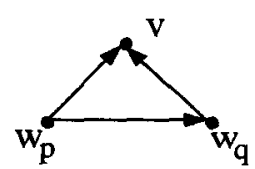

(a1)

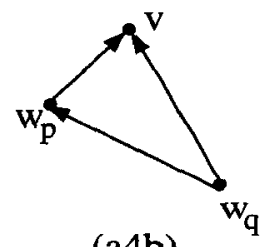

(a4b)

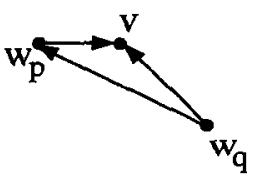

(a8a)

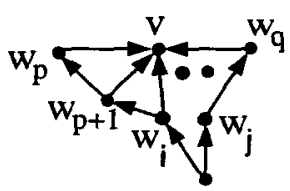

(b2a)

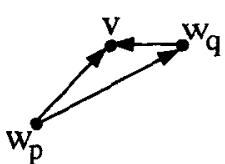

(a2)

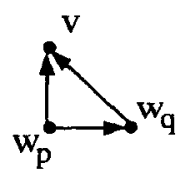

(a5)

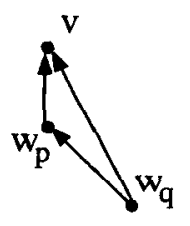

(a8b)

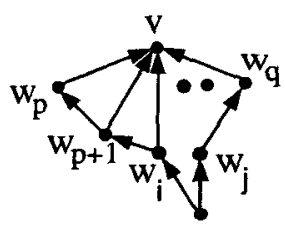

(b2b)

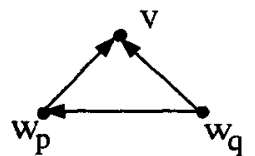

(a3)

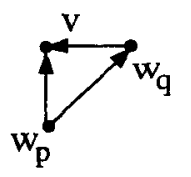

(a6)
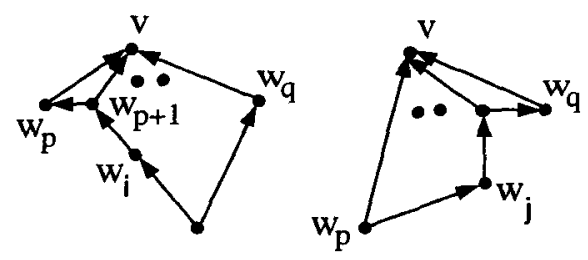

(b1)

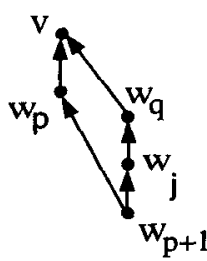

(b3)

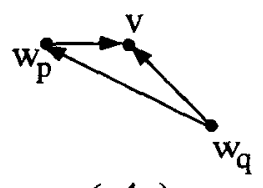

(a4a)

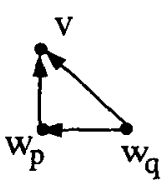

(a7)

Fig. 4. The placement rules.

We next describe the placement rules for $v$ when $\operatorname{deg}^{-}(v) \geq 3$. By Lemma 3.2 and the contour invariants, $v$ 's children $w_{p}, \ldots, w_{q}$ are embedded as a $V$-shaped polygonal line $P$. The vertex among $w_{p}$ and $w_{q}$ with the larger $y$-coordinate is called the high-end vertex of $v$. (If $y\left(w_{p}\right)=y\left(w_{q}\right)$, then both $w_{p}$ and $w_{q}$ are high-end vertices of $v$.) Let $\left(w_{i}, w_{i+1}\right)$ be the last downward edge in $P$. (If no such edge exists, let $w_{i}=w_{p}$.) Let $\left(w_{j-1}, w_{j}\right)$ be the first upward or vertical edge in $P$. (If no such edge exists, let $w_{j}=w_{q}$.) Note that the last downward edge in $P$ must appear before the first upward or vertical edge in $P$. So if $P$ has a downward edge, we have $x\left(w_{i}\right)<x\left(w_{j}\right)$. If $P$ has no downward edge, then $w_{i}=w_{p}$. By Lemma 3.3, the edge $\left(w_{p}, w_{p+1}\right)$ cannot be vertical. So we also have $x\left(w_{i}\right)<x\left(w_{j}\right)$. Thus we always have $x\left(w_{p}\right) \leq x\left(w_{i}\right)<x\left(w_{j}\right) \leq x\left(w_{q}\right)$. Consider 
any point $(x(v), y(v))$ such that:

(i) $x\left(w_{i}\right) \leq x(v)<x\left(w_{j}\right)$, if either $\left(w_{j-1}, w_{j}\right)$ or $\left(w_{j}, w_{j+1}\right)$ is vertical; $x\left(w_{i}\right) \leq$ $x(v) \leq x\left(w_{j}\right)$, if neither $\left(w_{j-1}, w_{j}\right)$ nor $\left(w_{j}, w_{j+1}\right)$ is vertical.

(ii) $y(v) \geq \max \left\{y\left(w_{p}\right), y\left(w_{q}\right)\right\}+1$, if the edge adjacent to the high-end vertex of $v$ is horizontal; $y(v) \geq \max \left\{y\left(w_{p}\right), y\left(w_{q}\right)\right\}$, if the edge adjacent to the high-end vertex of $v$ is not horizontal.

It is easy to see that such a point satisfies conditions (pr3), (pr4), and (pr5). In the following placement rules we specify $x(v), y(v)$ and show they satisfy conditions (i), (ii), (pr1) and (pr2). (Thus the placement requirements are satisfied.) Note that no shift operation is needed for any contour vertex.

Case (b1): $\operatorname{deg}^{-}(v) \geq 3$, the edge covered by $v$ and adjacent to the high-end vertex of $v$ is horizontal. (This includes the case when both $w_{p}$ and $w_{q}$ are high-end vertices of $v$, and either the edge adjacent to $w_{p}$ or the edge adjacent to $w_{q}$, or both, is horizontal.) $v$ is placed as in Fig. 4(b1) (two examples are shown): Let $y(v)=\max \left\{y\left(w_{p}\right), y\left(w_{q}\right)\right\}+1$ and determine $x(v)$ as follows:

- $v$ is stable. Let $x(v)=x\left(w_{i}\right)$. Then we have $x(v)=x\left(w_{i}\right)<x\left(w_{j}\right)$.

- $v$ is unstable. If $w_{i}=w_{p}$, let $x(v)=x\left(w_{p}\right)+1$. If $w_{i} \neq w_{p}$, let $x(v)=x\left(w_{i}\right)$. If $w_{i} \neq w_{p}$, then $x(v)=x\left(w_{i}\right)<x\left(w_{j}\right)$. If $w_{i}=w_{p}$, then $x(v)=x\left(w_{p}\right)+1$. By Lemma 3.3, $x\left(w_{p}\right)<x\left(w_{p+1}\right)<x\left(w_{p+2}\right)$. So the edges $e_{1}=\left(w_{p}, w_{p+1}\right)$ and $e_{2}=\left(w_{p+1}, w_{p+2}\right)$ cannot be vertical. If $e_{1}$ is upward, then $j=p+1$. In this case, $x(v) \leq x\left(w_{p+1}\right)=x\left(w_{j}\right)<x\left(w_{p+2}\right) \leq x\left(w_{q}\right)$. If $e_{1}$ is horizontal or downward, then $j \geq p+2$. In this case, $x(v) \leq x\left(w_{p+1}\right)<x\left(w_{p+2}\right) \leq x\left(w_{j}\right) \leq x\left(w_{q}\right)$.

$W$ is unchanged. $H$ is increased by at most 1 .

Case (b2a): $\operatorname{deg}^{-}(v) \geq 3$; the edge covered by $v$ and adjacent to the high-end vertex of $v$ is not horizontal; $w_{p}$ is the high-end vertex of $v$ (this includes the case when both $w_{p}$ and $w_{q}$ are high-end vertices of $\left.v\right)$; and $x\left(w_{j}\right) \geq x\left(w_{p}\right)+2 . v$ is placed as in Fig. 4(b2a): Let $y(v)=y\left(w_{p}\right)$ and $x(v)=\max \left\{x\left(w_{p}\right)+1, x\left(w_{i}\right)\right\}$. If $w_{i} \neq w_{p}$, then $x(v)=x\left(w_{i}\right)<x\left(w_{j}\right)$. If $w_{i}=w_{p}$, then $x(v)=x\left(w_{p}\right)+1<x\left(w_{j}\right) . W$ is unchanged. $H$ is unchanged.

Case (b2b): Same as in case (b2a). Figure 4(b2b) shows an alternative placement of $v$ : Let $y(v)=y\left(w_{p}\right)+1$, and $x(v)=\max \left\{x\left(w_{p}\right)+1, x\left(w_{i}\right)\right\}$. Similar to case (b2a), we can show $x(v)<x\left(w_{j}\right) . W$ is unchanged. $H$ is increased by at most 1 .

Case (b3): $\operatorname{deg}^{-}(v) \geq 3$; the edge covered by $v$ and adjacent to the high-end vertex of $v$ is not horizontal; $w_{p}$ is the high-end vertex of $v$ (this includes the case when both $w_{p}$ and $w_{q}$ are high-end vertices of $\left.v\right)$; and $x\left(w_{j}\right)=x\left(w_{p}\right)+1 . v$ is placed as in Fig. 4(b3). In this case the first edge $\left(w_{p}, w_{p+1}\right)$ covered by $v$ is downward and the edges $\left(w_{p+1}, w_{p+2}\right) \cdots\left(w_{j-1}, w_{j}\right)$ are vertical. So $v$ must be stable. (If $v$ was unstable, the edge $\left(w_{p+1}, w_{p+2}\right)$ would not be vertical by Lemma 3.3.) Let $y(v)=y\left(w_{p}\right)+1$ and $x(v)=x\left(w_{p}\right)$. Clearly $x(v)<x\left(w_{j}\right) . W$ is unchanged. $H$ is increased by at most 1 .

Case (b4): $\mathrm{deg}^{-}(v) \geq 3$; the edge covered by $v$ and adjacent to the high-end vertex of $v$ is not horizontal; $w_{q}$ is the only high-end vertex of $v . v$ is placed as in Fig. 4(b4): Let $y(v)=$ 
$y\left(w_{q}\right)$. If $v$ is stable, let $x(v)=x\left(w_{i}\right)$. If $v$ is unstable, let $x(v)=\max \left\{x\left(w_{p}\right)+1, x\left(w_{i}\right)\right\}$. By using the same argument as in case (b1), we can show conditions (i), (ii), (pr1), and (pr2) are satisfied. $W$ is unchanged. $H$ is unchanged.

This completes the description of the placement rules. In cases (a4), (a8), and (b2), two alternative placement rules are given. The two alternatives are chosen as follows: Suppose that the vertex $v=v_{k+1}(k+1<n-1)$ satisfies the conditions of rule (a4) (rules (a8) or (b2), respectively). Let $w_{p}, \ldots, w_{q}$ be the children of $v$ in the contour $C_{k}$. Let $u$ be the vertex that covers the edge $\left(w_{p}, v\right)$. Let $z_{1}, z_{2}, z_{3}$ be the first, the second, and the third child of $u$, respectively. If the following avoid-horizontal-forward-edge conditions hold, we must place $v$ by using rule (a4b) (rules ( $a 8 b$ ) or (b2b), respectively).

Definition 3.4. Avoid-horizontal-forward-edge conditions:

(1) $\operatorname{deg}^{-}(u) \geq 3$.

(2) $\left(w_{p}, v\right)$ is the last edge covered by $u$.

(3) either of the following two conditions hold:

(a) the edge $\left(z_{1}, z_{2}\right)$ is upward; or

(b) $z_{3} \neq v$, the edge $\left(z_{2}, z_{3}\right)$ is upward, and $y\left(z_{1}\right) \leq y\left(w_{p}\right)$.

Remark 1. Note that rules (a4a), (a8a), and (b2a) are the only rules that can create horizontal forward edges: By using rules (a4b), (a8b), and (b2b), this can be avoided. More precisely, suppose that $v$ satisfies the avoid-horizontal-forward-edge conditions. If $v$ is placed by using rules ( $44 \mathrm{a}),(\mathrm{a} 8 \mathrm{a})$, or ( $\mathrm{b} 2 \mathrm{a})$, the edge $\left(w_{p}, v\right)$ is horizontal and $v$ is not a peak point. This will cause $u$ to be a peak point (see Fig. 5(1)). If $v$ is placed by using rules $(\mathbf{a} 4 \mathrm{~b}),(\mathrm{a} 8 \mathrm{~b})$, or (b2b), the edge $\left(w_{p}, v\right)$ will be either upward or vertical, and $v$ will be a peak point. In this case $u$ will not be a peak point (see Fig. 5(2)). One might think that using rules ( $a 4 a)$, (a8a), and (b2a) is better than using rules (a4b), (a8b), and (b2b), since the placement of $v$ does not increase $H$. However, the placement of $u$ will increase $H$ in this case. So using rules (a4b), (a8b), and (b2b) merely shifts the contribution to $H$ from the vertex $u$ to the vertex $v$. Later, we will see that using rules (a4b), (a8b), and (b2b) allows us to prove the bound on $W+H$.

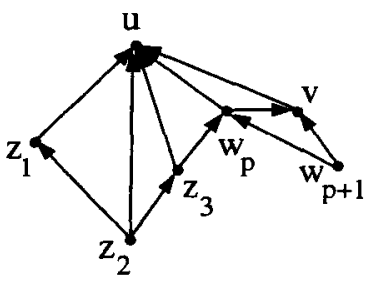

(1)

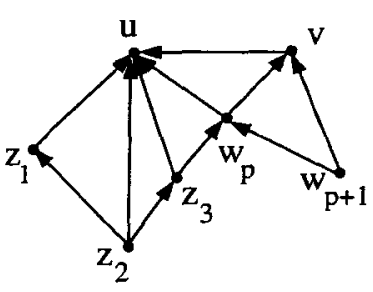

(2)

Fig. 5. The avoid-horizontal-forward-edge conditions. 
Remark 2. By the definition of $\pi_{\text {left }}$, the vertices $z_{1}, z_{2}, z_{3}$ are embedded before $v$ is embedded. So the avoid-horizontal-forward-edge conditions can be checked when $v$ is embedded.

For a vertex $v$ satisfying the conditions of rule (a4) (or (b2), respectively), if the avoid-horizontal-forward-edge conditions do not hold, then we place $v$ by using rule (a4a) (or (b2a), respectively). For a vertex $v$ satisfying the conditions of the rule (a8), if the avoid-horizontal-forward-edge conditions do not hold, $v$ is called a freelance vertex. A freelance vertex can be placed by using either rule (a8a) or (a8b). In the first case, $W$ is increased by at most 1 and $H$ is not changed. In the second case, $W$ is unchanged and $H$ is increased by at most 1 . We use this freedom to adjust the height and the width of the embedding as follows. Let $n_{\mathrm{r}}$ be the number of room-shift vertices. ( $n_{\mathrm{r}}$ can be computed when $\pi_{\text {left }}$ is constructed). Let $d=(n / 2-1)-n_{\mathrm{r}}$. (Later we show $d \geq 0$.) We place the first (at most) $d$ freelance vertices by using rule (a8a). All other (if any) freelance vertices are placed by using rule (a8b). This completes the description of our algorithm.

Example. Graph $G$ shown in Fig. 3 is embedded by using our algorithm. (Recall that the vertex $v_{n}=19$ is not embedded.) The rule used for each vertex is indicated in the figure. Vertex 9 is a freelance vertex and is placed by using rule (a8a). Vertex 11 is a (b2b) vertex since it satisfies the avoid-horizontal-forward-edge conditions. Although vertex 18 satisfies the avoid-horizontal-forward-edge conditions (because of vertex 19), since 19 need not be embedded, 18 is placed by using rule (b2a).

\section{Bounding the Grid Size}

In this section we prove the bounds on the size of the grid embedding produced by our algorithm, and complete the proof of Theorem 1.1. We first derive some equations on the grid size. Let $W$ and $H$ be the width and the height of the final grid. If a vertex $v$ is placed by using rule (ai) $(i=1,2,3,4 \mathrm{a}, 4 \mathrm{~b}, 5,6,7,8 \mathrm{a}, 8 \mathrm{~b})$ or rule $(\mathrm{b} i)(i=1,2 \mathrm{a}, 2 \mathrm{~b}, 3,4)$, we call $v$ an (ai) or a (bi) vertex, respectively. Let $a_{i}$ be the number of (ai) vertices and $b_{i}$ be the number of $(b i)$ vertices. A vertex that is the first one reaching a new $y$-coordinate is called a height-increasing vertex. A height-increasing vertex must be either an (ai) vertex (for $i=1,3,4 \mathrm{~b}, 5,7,8 \mathrm{~b}$ ), or a (bi) vertex (for $i=1,2 \mathrm{~b}, 3$ ). A height-increasing vertex of type (ai) or (bi) is called an $\left(\mathrm{a}^{\prime} i\right)$ or a $\left(\mathrm{b}^{\prime} i\right)$ vertex, respectively. Let $a_{i}^{\prime}$ and $b_{i}^{\prime}$ be the number of $\left(\mathrm{a}^{\prime} i\right)$ and $\left(\mathrm{b}^{\prime} i\right)$ vertices, respectively. We have

$$
H=1+a_{1}^{\prime}+a_{3}^{\prime}+a_{4 \mathrm{~b}}^{\prime}+a_{5}^{\prime}+a_{7}^{\prime}+a_{8 \mathrm{~b}}^{\prime}+b_{1}^{\prime}+b_{2 \mathrm{~b}}^{\prime}+b_{3}^{\prime} .
$$

If the placement of a vertex $v$ increases $W, v$ is called a width-increasing vertex. A width-increasing vertex must be an (a $i$ ) vertex (for $i=1,2,3,4 \mathrm{a}, 4 \mathrm{~b}, 8 \mathrm{a}$ ). Let $a_{i}^{\prime \prime}$ ( $i=1,2,3,4 \mathrm{a}, 4 \mathrm{~b}, 8 \mathrm{a}$ ) be the number of (ai) vertices that are not width-increasing. We have

$$
W=2+\left(a_{1}+a_{2}+a_{3}+a_{4 \mathrm{a}}+a_{4 \mathrm{~b}}+a_{8 \mathrm{a}}\right)-\left(a_{1}^{\prime \prime}+a_{2}^{\prime \prime}+a_{3}^{\prime \prime}+a_{4 \mathrm{a}}^{\prime \prime}+a_{4 \mathrm{~b}}^{\prime \prime}+a_{8 \mathrm{a}}^{\prime \prime}\right) .
$$

Since $v_{1}, v_{2}, v_{3}$ are neither (a $i$ ) nor (bi) vertices for any $i$, and $v_{n}$ is not embedded, we have

$$
a_{1}+a_{2}+a_{3}+a_{4 \mathrm{a}}+a_{4 \mathrm{~b}}+a_{5}+a_{6}+a_{7}+a_{8 \mathrm{a}}+a_{8 \mathrm{~b}}+b_{1}+b_{2 \mathrm{a}}+b_{2 \mathrm{~b}}+b_{3}+b_{4}=n-4 \text {. }
$$




\subsection{Bound on $H+W$}

From (1), (2), and (3), we have

$$
\begin{aligned}
W+H= & 3+\left(a_{1}+a_{2}+a_{3}+a_{4 \mathrm{a}}+a_{4 \mathrm{~b}}+a_{5}^{\prime}+a_{6}+a_{7}^{\prime}+a_{8 \mathrm{a}}+a_{8 \mathrm{~b}}^{\prime}\right. \\
& \left.\quad+b_{1}^{\prime}+b_{2 \mathrm{a}}+b_{2 \mathrm{~b}}^{\prime}+b_{3}^{\prime}+b_{4}\right) \\
& +\left(a_{1}^{\prime}+a_{3}^{\prime}+a_{4 \mathrm{~b}}^{\prime}\right)-\left(a_{6}+b_{2 \mathrm{a}}+b_{4}\right)-\left(a_{1}^{\prime \prime}+a_{2}^{\prime \prime}+a_{3}^{\prime \prime}+a_{4 \mathrm{a}}^{\prime \prime}+a_{4 \mathrm{~b}}^{\prime \prime}+a_{8 \mathrm{a}}^{\prime \prime}\right) \\
\leq & (n-1)+\left(a_{1}^{\prime}+a_{3}^{\prime}+a_{4 \mathrm{~b}}^{\prime}\right)-\left(a_{6}+b_{2 \mathrm{a}}+b_{4}\right) \\
& -\left(a_{1}^{\prime \prime}+a_{2}^{\prime \prime}+a_{3}^{\prime \prime}+a_{4 \mathrm{a}}^{\prime \prime}+a_{4 \mathrm{~b}}^{\prime \prime}+a_{8 \mathrm{a}}^{\prime \prime}\right) .
\end{aligned}
$$

Let $D$ denote the set of (a1), (a3), (a4b) vertices; $D^{\prime}$ the set of $\left(a^{\prime} 1\right),\left(a^{\prime} 3\right),\left(a^{\prime} 4 b\right)$ vertices; and $J$ the set of (a6), (b2a), (b4) vertices. Thus $\left|D^{\prime}\right|=a_{1}^{\prime}+a_{3}^{\prime}+a_{4 \mathrm{~b}}^{\prime}$ and $|J|=a_{6}+b_{2 \mathrm{a}}+b_{4}$. Note that a vertex in $D^{\prime}$ may increase both $W$ and $H$ by 1 , while a vertex in $J$ increases neither $W$ nor $H$. We will show $\left|D^{\prime}\right| \leq|J|$. Define:

$K=\left\{v \mid v\right.$ is unstable and the edge $\left(w_{p}, v\right)$ is upward, where $w_{p}$ is the leftmost child of $v$ \}.

Note that $D^{\prime} \subseteq D \subseteq K$. For each $v \in K$, we define a sequence of vertices $S(v)=\left(x_{0}=\right.$ $v, x_{1}, \ldots, x_{k}, u$ ) (possibly $k=0$ ) such that:

(1) For each $i(1 \leq i \leq k), x_{i}$ is an (a2) vertex and covers the edge $\left(w_{p}, x_{i-1}\right)$.

(2) $u$ is either an (a6), (b4), (b2a), or (b2b) vertex and covers the edge $\left(w_{p}, x_{k}\right)$.

$S(v)$ is defined as follows. Start with $S=\left(x_{0}=v\right)$. Suppose that $x_{i}$ has been defined. Let $u$ be the vertex that covers the edge $\left(w_{p}, x_{i}\right)$, where $w_{p}$ is the leftmost child of $x_{i}$. There are three cases:

Case 1: $\operatorname{deg}^{-}(u)=2$ and $u$ is unstable. Then define $x_{i+1}=u$, and continue. Note that the edge $\left(w_{p}, x_{i}\right)$ is upward. By our placement rules, $x_{i+1}$ must be an (a2) vertex.

Case 2: $\operatorname{deg}^{-}(u)=2$ and $u$ is stable. Let $k=i$ and $u$ be the last vertex of $S(v)$ and we are done. Note that since $\left(w_{p}, x_{k}\right)$ is upward, $u$ must be an (a6) vertex.

Case 3: $\operatorname{deg}^{-}(u) \geq 3$. Let $k=i$ and $u$ be the last vertex of $S(v)$ and we are done. Since $u$ covers the edge $\left(w_{p}, x_{k}\right)$ and $x_{k}$ is unstable, there are two subcases:

Case 3A: $\operatorname{ind}_{u}\left(x_{k}\right)=2$. Since the edge $\left(w_{p}, x_{k}\right)$ is upward, $u$ is forward-oriented. (See Fig. 6(1)). By the avoid-horizontal-forward-edge conditions and the placement rules, the last edge covered by $u$ must be either upward or vertical. Thus, $u$ is placed as a (b4) vertex.

Case 3B: ind $_{u}\left(x_{k}\right)=3$ and $u$ is unstable. Let $e_{1}=\left(t_{1}, w_{p}\right)$ and $e_{2}=\left(t_{2}, t_{3}\right)$ be the first and the last edge covered by $u$ (see Fig. 6(2)).

- Suppose $\mathrm{deg}^{-}(u)>3$ and $y\left(t_{1}\right) \leq y\left(t_{2}\right)$. By the avoid-horizontal-forward-edge conditions, $e_{2}$ is upward or vertical and $t_{3}$ is the only high-end vertex of $u$. So $u$ is placed as a (b4) vertex.

- Suppose $\operatorname{deg}^{-}(u)>3$ and $y\left(t_{1}\right)>y\left(t_{2}\right)$. If $e_{2}$ is horizontal, then $t_{1}$ is the only high-end vertex of $u$ and the edge $e_{1}$ is not horizontal. If $e_{2}$ is upward, then neither 


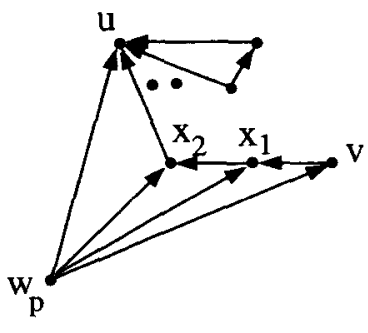

(1)

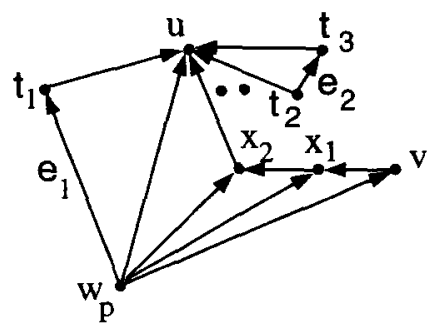

(2)

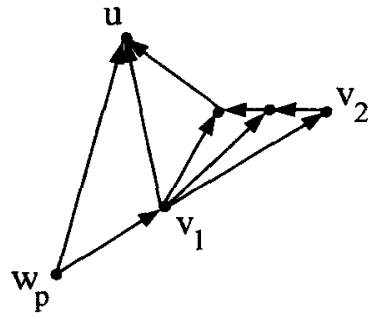

(3)

Fig. 6. The last vertex $u$ in the sequence $S(v)$.

$e_{1}$ nor $e_{2}$ is horizontal. So $u$ is placed either as a (b2a), (b2b), (b4) vertex. (Since both the first and the second edge covered by $u$ are not vertical, rule (b3) does not apply.)

- Suppose $\mathrm{deg}^{-}(u)=3$. Then $\left(w_{p}, x_{k}\right)=\left(t_{2}, t_{3}\right)$. Depending on which of $t_{1}$ and $t_{3}$ is the high-end vertex of $u, u$ is either a (b2a), (b2b), or a (b4) vertex.

Note that in $S(v)=\left\{x_{0}=v, x_{1}, \ldots, x_{k}, u\right\}$, the vertices $x_{1}, \ldots, x_{k}$ (if any) are uniquely determined by $v$. On the other hand, a vertex $u$ can be the last vertex of $S\left(v_{1}\right)$ and $S\left(v_{2}\right)$ for two distinct vertices $v_{1}, v_{2} \in K$, where $u$ satisfies case $3 \mathrm{~A}$ for $S\left(v_{1}\right)$ and case 3B for $S\left(v_{2}\right)$. (See Fig. 6(3).)

We construct a directed forest $F$ such that the following hold:

- The node set of $F$ is a subset of the vertices of $G^{+}$.

- The set of leaf nodes in $F$ is the set $D$.

- Each nonleaf internal node of $F$ is a (b4) vertex, and has exactly two children.

- Each root node of $F$ is either a (b2b) vertex or in $J$, and has exactly one child.

The forest $F$ is constructed as follows. We keep a set $Q \subseteq K$. For each $v \in Q$, we find the sequence $S(v)$ and define parent $(v)$ to be the last vertex $u$ of $S(v)$. Note that $u$ is either in $J$ or a (b2b) vertex. When a vertex $v$ is put in $Q$, parent $(v)$ is identified. At any moment, for each vertex $v \in Q$, parent $(v)$ is the root of a tree. Initially, let $Q=D$.

By the remark above, at most two vertices $v_{1}, v_{2}$ in $Q$ can have the same parent $u$. In this case $u$ must be unstable and the edge $\left(w_{p}, u\right)$ (where $w_{p}$ is the leftmost child of $u$ ) is upward. (See Fig. 6(3).) So $u$ is a (b4) vertex and is also in $K$. In this case we remove $v_{1}, v_{2}$ from $Q$ and put $u$ into $Q$.

Repeat this process until all the vertices in $Q$ have distinct parents. At this point, the parents of the vertices in $Q$ are the roots of the trees of the forest $F$ to be constructed. It is easy to check that $F$ satisfies all the above conditions.

Figure 7 shows an example of this construction. A portion of graph $G$ and its corresponding forest $F$ is shown. Vertices $v_{1}, v_{2}, v_{3}$ are in $D$. Root $u_{3}$ is a (b2b) vertex.

Consider a tree $T$ in $F$. Let $\operatorname{Leaf}(T)$ and $\operatorname{Int}(T)$ be the number of leaf nodes and internal nodes in $T$, respectively. Clearly, $\operatorname{Leaf}(T)=\operatorname{Int}(T)$. Let $D(T), D^{\prime}(T)$, and $J(T)$ be the number of nodes in $T$ that are in $D, D^{\prime}$, and $J$, respectively. We show $D^{\prime}(T) \leq J(T)$. 


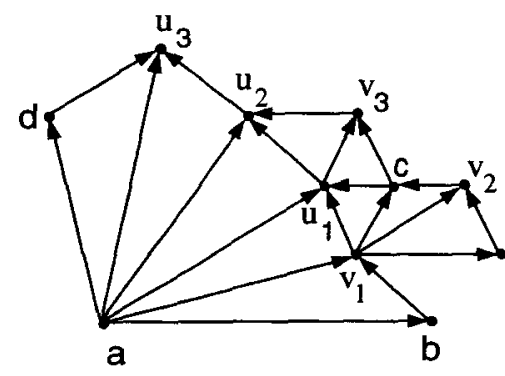

G

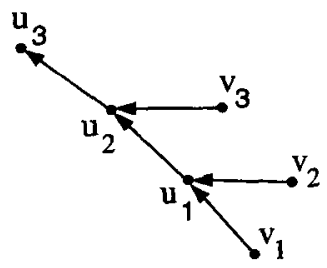

$\mathrm{F}$

Fig. 7. The construction of the forest $F$.

If the root of $T$ is in $J$, then $D^{\prime}(T) \leq D(T)=$ Leaf $(T)=\operatorname{lnt}(T)=J(T)$.

Suppose the root of $T$ is a (b2b) vertex. Let $v$ be the leaf node in $T$ that has the smallest $y$-coordinate among all leaf nodes in $T$. Then $v$ is not a height-increasing vertex. (See Fig. 7. Vertex $v_{1}$ has the smallest $y$-coordinate. It is not height-increasing, because there is a vertex $z$ such that $y(z)=y\left(v_{1}\right)$ and $z \prec_{\text {left }} d \prec_{\text {left }} v_{1}$ ). Thus $v$ is in $D$ but not in $D^{\prime}$. Hence, $D^{\prime}(T) \leq D(T)-1=\operatorname{Leaf}(T)-1=\operatorname{Int}(T)-1=J(T)$.

Since each vertex in $D^{\prime}$ corresponds to a distinct leaf node in $F$ and $D^{\prime}(T) \leq J(T)$ holds for every tree $T$ in $F$, we have $|D|^{\prime} \leq|J|$. From inequality (4), this gives

$$
W+H \leq(n-1)-\left(a_{1}^{\prime \prime}+a_{2}^{\prime \prime}+a_{3}^{\prime \prime}+a_{4 \mathrm{a}}^{\prime \prime}+a_{4 \mathrm{~b}}^{\prime \prime}+a_{8 \mathrm{a}}^{\prime \prime}\right) \leq(n-1) .
$$

\subsection{Bound on the Width W}

We first bound $n_{\mathrm{r}}$ (the number of room-shift vertices in $G^{+}$). Each room-shift vertex $v$ is associated with a vertex $\operatorname{dom}(v)$. It has been shown in [4] that, for two distinct room-shift vertices $v_{i}$ and $v_{j}, \operatorname{dom}\left(v_{i}\right) \neq \operatorname{dom}\left(v_{j}\right)$. From the definition of the dominator, it is easily seen that only the forward-oriented vertices and $v_{n}$ can be dominators of room-shift vertices. By our assumption on canonical ordering, we have $n_{\mathrm{f}} \leq n_{\mathrm{b}}$. Hence:

$$
n_{\mathrm{r}}=a_{1}+a_{2}+a_{3}+a_{4 \mathrm{a}}+a_{4 \mathrm{~b}} \leq n_{f}+1 \leq(n-4) / 2+1=n / 2-1 .
$$

Thus $d=(n / 2-1)-n_{\mathrm{r}} \geq 0$. Our algorithm places at most $d$ freelance vertices by using the rule (a8a). So $a_{8 \mathrm{a}} \leq d$. From (2), we have

$$
W \leq 2+\left(a_{1}+a_{2}+a_{3}+a_{4 \mathrm{a}}+a_{4 \mathrm{~b}}\right)+a_{8 \mathrm{a}} \leq 2+n_{\mathrm{r}}+(n / 2-1)-n_{\mathrm{r}}=n / 2+1 .
$$

\subsection{Bound on the Height $H$}

First suppose the number of freelance vertices is at least $d=(n / 2-1)-n_{\mathrm{r}}$. Then our algorithm places $a_{8 \mathrm{a}}=d$ of them by using rule (a8a). Thus

$$
a_{1}+a_{2}+a_{3}+a_{4 \mathrm{a}}+a_{4 \mathrm{~b}}+a_{8 \mathrm{a}}=n_{\mathrm{r}}+d=n / 2-1 .
$$




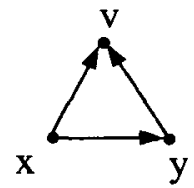

(1)

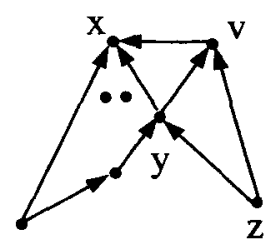

(2)

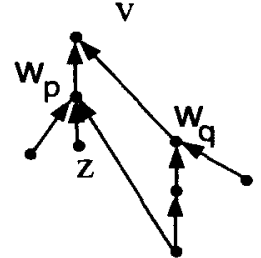

(3)

Fig. 8. Definition of the mate vertices.

From this, (2), and inequality (5), we have

$$
\begin{aligned}
H & \leq(n-1)-\left(a_{1}^{\prime \prime}+a_{2}^{\prime \prime}+a_{3}^{\prime \prime}+a_{4 \mathrm{a}}^{\prime \prime}+a_{4 \mathrm{~b}}^{\prime \prime}+a_{8 \mathrm{a}}^{\prime \prime}\right)-W \\
& =(n-1)-\left(2+a_{1}+a_{2}+a_{3}+a_{4 \mathrm{a}}+a_{4 \mathrm{~b}}+a_{8 \mathrm{a}}\right) \\
& =(n-1)-(n / 2+1)=n / 2-2<2(n-2) / 3 .
\end{aligned}
$$

Next suppose the number of freelance vertices is less than $d$. Then no freelance vertices are placed by using rule ( $\mathrm{a} 8 \mathrm{~b})$. So all $(\mathrm{a} 8 \mathrm{~b}$ ) vertices satisfy the avoid-horizontalforward-edge conditions.

Let $A$ denote the set of $\left(a^{\prime} 1\right),\left(a^{\prime} 3\right),\left(a^{\prime} 5\right),\left(a^{\prime} 7\right)$, and $\left(b^{\prime} 1\right)$ vertices; $B$ the set of $\left(a^{\prime} 4 b\right)$, $\left(a^{\prime} 8 b\right),\left(b^{\prime} 2 b\right)$ vertices; and $C$ the set of $\left(b^{\prime} 3\right)$ vertices. From (1), we have

$$
H=1+|A|+|B|+|C| .
$$

For each $v \in A \cup B \cup C$, we define a vertex mate ( $v$ ) as follows.

Consider a vertex $v \in A . v$ covers a horizontal edge $(x, y)$ with $y$-coordinate $y(v)-1$. (See Fig. 8(1)). At most one of $x$ and $y$ can be a height-increasing vertex. Define mate $(v)$ to be the vertex among $x$ and $y$ that is not a height-increasing vertex.

Consider a vertex $v \in B$. Let $y$ and $z$ be the first and the second child of $v$. Let $x$ be the vertex that covers the edge $(y, v)$. (See Fig. 8(2).) By Remark $1, v$ is a peak point and the edge $(x, v)$ is horizontal. So $x$ is not a height-increasing vertex. Define mate $(v)=x$.

Consider a vertex $v \in C$. Let $w_{p}$ and $w_{q}$ be the leftmost and rightmost child of $v$, respectively. (See Fig. 8(3).) By rule (b3), we have $y\left(w_{p}\right) \geq y\left(w_{q}\right)$. By the definition of the leftmost canonical ordering $\pi_{\text {left }}$, we have $w_{p} \prec_{\text {left }} w_{q}$. Thus there is a vertex $z$ such that $z \prec_{\text {left }} w_{p} \prec_{\text {left }} w_{q}$ and $y(z)=y\left(w_{q}\right)$. So $w_{q}$ is not a height-increasing vertex. Define mate $(v)=w_{q}$.

Define: Mate $=\{$ mate $(v) \mid v \in A \cup B \cup C\}$. From the definition, it is easy to check that:

- Mate $\cap(A \cup B \cup C)=\emptyset$. This is because that all vertices in $A \cup B \cup C$ are height-increasing while the vertices in Mate are not height-increasing.

- A vertex $x$ can be the mate vertex for at most two vertices: one $v$ with $y(v)=y(x)$ (namely $v \in B$ ) and another $v^{\prime}$ with $y\left(v^{\prime}\right)>y(x)$ (namely $v^{\prime} \in A$ or $v^{\prime} \in C$ ).

Hence each vertex in Mate corresponds to at most two vertices in $A \cup B \cup C$. Thus:

$$
|A|+|B|+|C| \leq 2 \mid \text { Mate } \mid \text {. }
$$


The vertices $v_{1}, v_{2}, v_{3}, v_{n}$ are neither in $A \cup B \cup C$ nor in Mate. Thus:

$$
|A|+|B|+|C|+\mid \text { Mate } \mid \leq(n-4) .
$$

So $|A|+|B|+|C| \leq 2(n-4) / 3$. From (1), this gives

$$
H=1+(|A|+|B|+|C|) \leq 1+2(n-4) / 3<2(n-2) / 3 .
$$

Recall that $G^{+}$has $n$ vertices, and the original graph $G$ has $n-1$ vertices. So the bounds in inequalities (5), (6), (7), and (8) imply the bounds on grid size stated in Theorem 1.1.

\subsection{Implementation of the Algorithm}

The extended graph $G^{+}$can be constructed from $G$ in linear time. The canonical ordering $\pi$ in Theorem 3.1 can be computed in $O(n)$ time [14]. The leftmost canonical ordering $\pi_{\text {left }}$ can be computed from $\pi$ in linear time. After $\pi_{\text {left }}$ is known, the algorithm can be implemented by using the method in [4]. We only note that, as stated in Remark 2, since $\pi_{\text {left }}$ is used, when a vertex $v$ is added, the conditions of the placement rules (including the avoid-horizontal-forward-edge conditions) can be checked in constant time. So the algorithm takes linear time. This completes the proof of Theorem 1.1.

\section{Conclusions}

We have shown that every 4-connected plane graph with at least four vertices on its external face has a straight line embedding on a $W \times H$ grid such that $W+H \leq n$, $W \leq(n+3) / 2$ and $H \leq 2(n-1) / 3$. The embedding can be constructed in linear time.

Consider the graph $G$ shown in Fig. 9(1). The square $S_{1}$ can be embedded in a $1 \times 1$

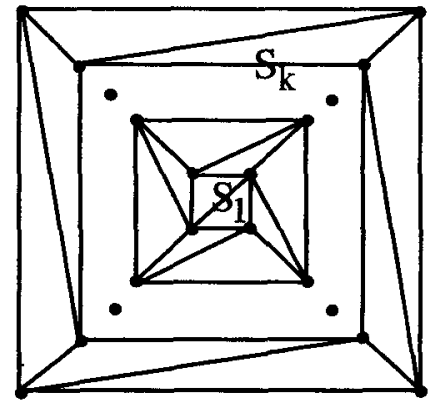

(1)

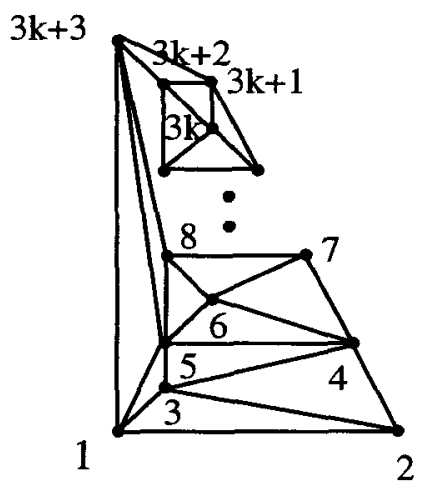

(2)

Fig. 9. Graphs that require large grids. 
grid. It is easy to show that after $S_{k}$ has been embedded, we need at least two more $x$-coordinates and two more $y$-coordinates to embed the square $S_{k+1}$. Hence any straight line embedding of $G$ must use a grid of size at least $(n / 2-1) \times(n / 2-1)$. We conjecture that every 4-connected plane graph with at least four vertices on its external face can be embedded in an $n / 2 \times n / 2$ grid. Our algorithm cannot achieve this bound. An example is shown in Fig. 9(2). This graph has $3 k+2$ vertices connected as shown, where the extra vertex $v_{n}=3 k+3$ is added and adjacent to every vertex on the external face. Because of the avoid-horizontal-forward-edge conditions, our algorithm places vertex 4 by rule (a4b) and vertices $3 i+1(2 \leq i \leq k)$ by rule (a8b). The size of the embedding is $(k+2) \times 2 k$. (It is easy to embed this graph in an $n / 2 \times n / 2$ grid, though.) It will be interesting to either prove or disprove the above conjecture.

\section{References}

1. J. Bondy and U. Murty, Graph Theory with Applications, North-Holland, Amsterdam, 1976.

2. N. Chiba, T. Yamanouchi, and T. Nishizeki, Linear algorithms for convex drawings of planar graphs, in Progress in Graph Theory, J. A. Bondy and U. S. R. Murty (eds.), 1982, pp. 153-173.

3. M. Chrobak and G. Kant, Convex grid drawings of 3-connected planar graphs, Technical Report RUUCS-93-45, Department of Computer Science, Utrecht University, 1993.

4. M. Chrobak and S. Nakano, Minimum-width grid drawings of planar graphs, Proc. Workshop on Graph Drawing, 1994, pp. 104-1 10, Lecture Notes in Computer Science, Vol. 894, Springer-Verlag, Berlin.

5. M. Chrobak and T. Payne, A linear time algorithm for drawing planar graphs on a grid, Technical Report UCR-CS-89-1, Department of Mathematics and Computer Science, University of California at Riverside, 1989.

6. P. Eades and R. Tamassia, Algorithms for automatic graph drawing: an annotated bibliography, Technical Report, Department of Computer Science, Brown University, 1993.

7. S. Even, Graph Algorithms, Computer Science Press, Rockville, MD, 1979.

8. I. Fáry, On straight line representation of planar graphs, Acta. Sci. Math. (Szeged) 11 (1948), 229-233.

9. H. de Fraysseix, J. Pach, and R. Pollack, Small sets supporting straight-line embeddings of planar graphs, Proc. 20th Annual ACM Symp. on Theary of Computing, 1988, pp. 426-433.

10. H. de Fraysseix, J. Pach, and R. Pollack, How to draw a planar graph on a grid, Combinatorica 10 (1990), 41-51.

11. M. Fürer, X. He, M. Y. Kao, and B. Raghavachari, $O(n \log \log n)$-work parallel algorithms for straight line grid embeddings of planar graphs, Proc. 4th Annual ACM Symp. on Parallel Algorithms and Architectures, 1992, pp. 410-419. Complete version appeared in SIAM J. Discrete Math. 7(4) (1994), 632-646.

12. G. Kant, Drawing planar graphs using the lmc-ordering, Proc. 33th Annual IEEE Symp. on Foundations of Computer Science, Pittsburgh, 1992, pp. 101-110.

13. G. Kant, Algorithms for drawing planar graphs, Ph.D. Dissertation, Department of Computer Science, University of Utrecht, 1993.

14. G. Kant and X. He, Two algorithms for finding rectangular duals of planar graphs, Proc. 19th Workshop on Graph-Theoretic Concepts in Computer Science, Utrecht, June 16-18, 1993, pp. 396-410, Lecture Notes in Computer Science, Vol. 790, Springer-Verlag, Berlin. To appear in Theoretical Computer Science.

15. F. P. Preparata and R. Tamassia, Fully dynamic techniques for point location and transitive closure in planar structures, Proc. 29th Annual IEEE Symp. on Foundations of Computer Science, 1988, pp. 558-567.

16. R. C. Read, A new method for drawing a planar graph given the cyclic order of the edges at each vertex, Congr. Numer. 56 (1987), 31-44.

17. P. Rosenstiehl and R. Tarjan, Rectilinear planar layouts and bipolar orientations of planar graphs, Discrete Comput. Geom. 1 (1986), 343-353.

18. W. Schnyder, Embedding planar graphs on the grid, Abstracts Amer. Math. Soc. 9 (1988), 268.

19. W. Schnyder, Planar graphs and poset dimension, Orders 5 (1989), 323-343. 
20. W. Schnyder, Embedding planar graphs on the grid, Proc. 1st Annual ACM-SIAM Symp. on Discrete Algorithms, 1990 , pp. 138-147.

21. W. Schnyder and W. Trotter, Convex drawings of planar graphs, Abstracts of Amer. Math. Soc. 13(5) (1992), 92T-05-135.

22. S. K. Stein, Convex maps, Proc. Amer. Math. Soc. 2 (1951), 464-466.

23. W. T. Tutte, How to draw a graph, Proc. London Math. Soc. 13 (1963), 743-768.

24. K. Wagner, Bemerkungen zum Vierfarben problem, Jahresber. Deutsch Math.-Verein. 46 (1936), 26-32.

Received March 30, 1995, and in revised form January 3, 1996. 